\title{
Adaptability Measures for Wheat Genotypes Evaluated under Northern Hills Zone of Country for Irrigated Timely Sown Conditions
}

\author{
Ajay Verma*1 ${ }^{* 1}$ J. Crossa ${ }^{1}$, M. Vargas ${ }^{1}$, A.K. Joshi ${ }^{2}$ and G.P. Singh ${ }^{3}$ \\ ${ }^{1}$ Senior Biometrician \& Senior Statistician, CIMMYT, Mexico \\ ${ }^{2}$ Director, CIMMYT Regional Office for Asia, New Delhi, India \\ ${ }^{3}$ ICAR-Indian Institute of Wheat \& Barley Research, Post Bag \# 158 Agrasain Marg, Karnal, Haryana, India \\ *Corresponding author : ajay.verma1@icar.gov.in (ORCID ID: 0000-0001-9255-6134)
}

Paper No. 810

Received: 23-10-2019

Revised: $15-01-2020$

Accepted: 25-02-2020

\begin{abstract}
Wheat genotypes were evaluated under multi environment trials for Northern Hills Zone of India to study the adaptability performance. Genotypes HS612, HS507 and HPW430 were of high yield and better adaptability by analytic measures of adaptability based on BLUP values during 2015-16. Two interaction principal components, accounted for $89.9 \%$ of total GxE interaction sum of squares in biplot analysis. HPW428, HS613, VL2020, VL2024 had specific adaptations to Almora and Malan while HS616, HPW423, HPW430, VL2021, HPW426 expressed for Shimla and Khudwani locations. Wheat genotypes HS612, HS507 and HPW430 were cited by analytic measures as per BLUE values. HPW429, HS613, VL2020, VL2024 had specific adaptations to Almora and Malan while HS616, HS618, HPW425, HPW426, HPW430, VL2023, VL2021, HPW426 observed for Shimla and Khudwani. Second year (2017-18) had seen high yield and better adaptability of HS631, HS632, VL2030, VL2025 genotypes as per BLUP values. Biplot analysis expressed specific adaptations of HPW429, HS613, VL2020, VL2024 to Almora and Malan locations. BLUE values based measures showed high yield and better adaptability of HS631, HS632, VL2030, VL2025 genotypes. Biplot analysis while utilizing 79.5\% of total GxE interaction sum of squares exhibited specific adaptations of HPW446, VL907, HS632, VL2025, VL2030 to Almora and Shimla. Stratification of wheat genotypes as per BLUP values was more efficient than that by BLUE. Biplot analysis exhibited more of GxE interactions sum of squares by first two significant principal components based on BLUP as compared to BLUE values.
\end{abstract}

\section{Highlights}

( Adaptability of wheat genotypes were studied by analytic measures based on random and fixed effects of genotypes. Further clustering pattern were studied among measures based on biplot analysis.

Keywords: MET, BLUP, BLUE, GAI, HMGV, RPGV, HMRPGV, Biplot analysis

Wheat improvement programs conduct multi environment trials (MET) for estimation of main of genotypes, environments and genotype $x$ environment interactions (Crespo et al. 2017). The cross over GxE interactions hampers the real yield potential and stable performance of genotypes as relative ranking of genotypes change across locations (Elesandro et al. 2017). Though, analysis of variance (ANOVA) technique estimates the influence of the different factors underlying phenotypic variation (Gogel et al. 2018). More over all factors are considered as of fixed effects along with homogeneity of variance and the same covariance for all pairs of genotypes. These assumptions are often inappropriate and unreliable (Friesen et al. 2016). The mixed model method considers fixed and random effects in commonly used experimental designs and correctly estimates 
genotype effect $(G)$ and genotype $\times$ environment $(G \times E)$ interaction effects using an appropriate variance-covariance structure (Nuvunga et al. 2018). The procedures based on restricted maximum likelihood/best linear unbiased prediction (REML/ BLUP) have proven to be effective in assessing genotypic performance (Santos et al. 2015). The statistical approach based on restricted maximum likelihood (REML) provides efficient estimates of variance components and genetic parameters (Smith and Cullis, 2018). Methods of mixed models have been gaining more and more space in the statistical evaluation of genotypes in plant-breeding trials, for crops, such as maize (Mendes et al. 2012; Baretta et al. 2016), corn (Oliveira et al. 2017), and cotton (Moiana et al. 2014) and cowpea (Torres et al. 2015). Prime objective of the study was to compare the adaptability performance of wheat genotypes as per their BLUP and BLUE values by analytic measures.

\section{MATERIALS AND METHODS}

Northern hills zone encompasses the hilly terrain of Northern region extending from Jammu \& Kashmir to North Eastern States. NHZ comprises J\&K (except Jammu and Kathua distt.); Himachal Pradesh (except Una and Paonta Valley); Uttarakhand (except Tarai area); Sikkim, hills of West Bengal and North Eastern states. Advanced wheat genotypes were evaluated in field trials at major locations of the zone during cropping season's viz. 201516 and 2017-18 as details are reflected in tables 1 \&2 for ready reference. Randomized block design with three replications were used for research field trials and recommended agronomical practices had followed to harvest good crop. More over yield were further analysed as per recent analytic adaptability measures.

Simple and effective measure for adaptability is based on the relative performance of genetic values (PRVG) across environments. MHVG method (harmonic mean of genetic values) as based on the harmonic mean of the genotypic values considered the yield \& stability. The lower the standard deviation of genotypic performance across environments, the greater is the harmonic mean of genotypes. For the use of mixed models, the simultaneous analysis of stability, adaptability and yield based on the harmonic mean of the relative performance of the genotypic values (MHPRVG).
The MHPRVG combines the methods PRVG and MHVG, simultaneously. Consequently, the selection for higher values of the harmonic mean results in selection for both yield and stability (Resende and Duarte, 2007).

$P R V G_{i j}=V G_{i j} / V G_{i}$

$M H V G_{i}=$ Number of environments $/ \sum_{i=1}^{k} \frac{1}{X_{i}}$ $M H P R V G_{i}=$ Number of environments $/ \sum_{j=1}^{k} \frac{1}{P R V G_{i j}}$

$V G_{i j}$ is the genotypic value of the $i$ genotype, in the $j$ environment, expressed as a proportion of the average in this environment. $P R V G$ and $M H P R V G$ values were multiplied by the general mean (GM) to have results in the same magnitude as of the average wheat yield in order to facilitate interpretation (Verardi et al. 2009). Estimation of the variance components were carried out by using residual maximum likelihood (REML) along with estimation/ prediction of the fixed as well as random effects by ASReml-R package. Mohammadi \& Amri, 2008 defined geometric adaptability index (GAI) to evaluate the adaptability of genotypes as GAI =

$$
\sqrt[n]{\prod_{k=1}^{n} \bar{X}_{k}}
$$

in which $\bar{X}_{1}, \bar{X}_{2}, \bar{X}_{3}, \ldots m$ are the mean yields of the first, second and $m^{\text {th }}$ genotype across environments and $n$ is number of environments. Genotypes with higher values of GAI are desirable.

\section{RESULTS AND DISCUSSION}

\section{First year (2015-16) based on BLUP}

\section{Analytic measures}

Average yield of genotypes as per BLUP values identified HS612, HS507 and HPW430 as of high yield with better adaptations while HS615 \& UP2952 expressed low yield. Harmonic mean ranked genotypes as HS612, HPW430 and VL2024 for better adaptation at the same time pointed out suitability of HS615 \& HS617 for specific adaptations (Table $3)$. Least values of standard error reflected the consistent performance of VL2019, UP2952, VL907 for considered location of this zone. Higher values of Geometric Adaptability Index selected HS612, HPW430,VL2023 as suitable wheat genotypes. PRVG 
as well as $\mathrm{PRVG}^{*} \mathrm{GM}$ pointed towards HS612, HS507 and HPW430 for the better adaptable behavior and HS615 \& HS617 of low adaptability under irrigated timely sown conditions for Northern Hills Zone. Most cited analytic measures HMPRVG and HMPRVG*GM marked HS612, HS507 and HPW430 as of high yield and better adaptability across major locations of this zone while HS615 \& HS617 for low degree of adaptation. An overall agreement has been observed among analytic measures PRVG, MHVG, MHPRVG, GAI and average yield for the classification of wheat genotypes (Table 3) (de Pelegrin et al. 2017). Variations among ranks of genotypes as per analytic adaptability measures observed as per various locations to highlight presence of cross over interactions effects for studied genotypes (Table 4).

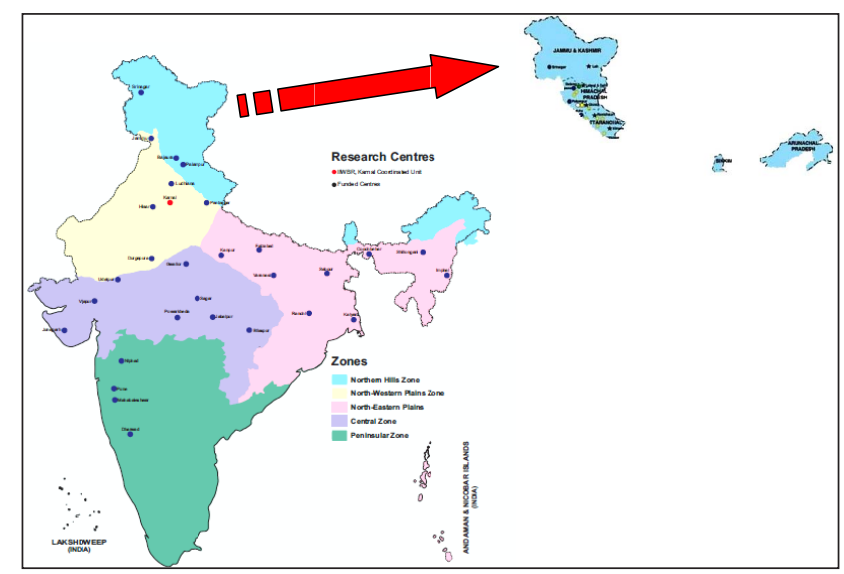

Fig. 1: Agro climatics zones for wheat cultivation in country

\section{Biplot analysis}

First two highly significant Interaction Principal Components expressed stable yield of HS618, UP2953, HS613, HPW431 and HPW428 genotypes in Biplot analysis. HS612, VL2019 and HS615 would be good for specific adaptations. Two interaction principal components, accounted for $89.9 \%$ of total $\mathrm{G} \times \mathrm{E}$ interaction sum of squares (Fig. 2). Shimla would be suitable environment for stable yield of genotypes. Environments Khudwani and Almora observed as larger contributor to the G $\times \mathrm{E}$ interactions, because as positioned relatively away from the origin. Biplot presentations were considered more stable; however, the greater the distance from the source the lower the stability related to the grain yield character; these effects are due to the nature of the $G \times E$ interaction (Yan and Kang 2003). Genotypes and environments placed in proximity have positive associations as these observations would enable to identify specific adaptations of the genotypes. HPW428, HS613, VL2020, VL2024 had specific adaptations to Almora and Malan while HS616, HPW423, HPW430, VL2021, HPW426 for Shimla and Khudwani. Location Almora with Malan, Khudwani with Shimla would show similar performance of genotypes as acute angles expressed among rays connecting these environments. Almora had an obtuse angle with Khudwani this would express opposite performance of genotypes i.e. HPW426 \& HPW428 will not be of choice for Almora. Standard error had been observed at distant from others analytic measures.

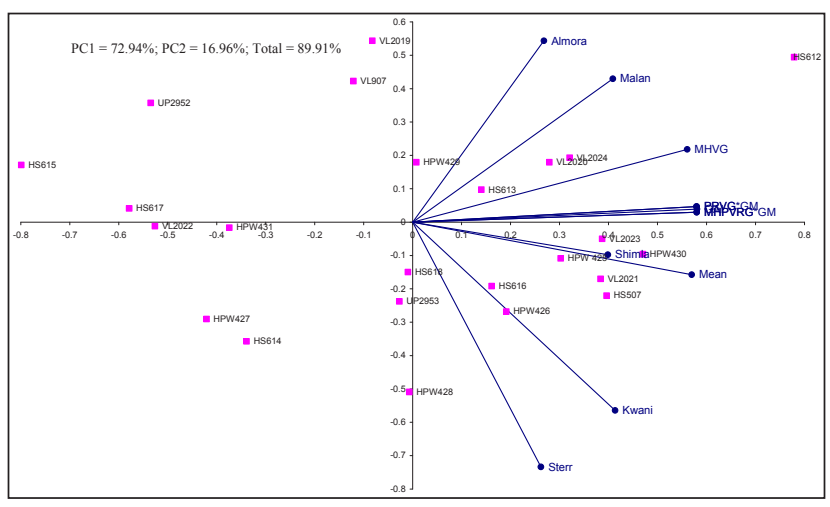

Fig. 2: Biplot analysis of genotypes vis-à-vis environments based on BLUP (2015-16)

\section{First year (2015-16) based on BLUE}

\section{Analytic measures}

Mean yield of wheat genotypes based on BLUE values selected HS612, HPW430 and HS507as of high yield with better adaptations while HS615 \& UP2952 achieved low yield. Ranking of genotypes based on harmonic mean selected HS612, HPW430 and VL2024 as better adapted genotypes along with suitability of HS615 \& HS617 for specific adaptations (Table 5). Lower values of standard error associated with the consistent performance of VL2019, UP2952, VL907 for considered locations of this zone. Higher values of Geometric Adaptability Index pointed towards HS612, HPW430, VL2023 as suitable wheat genotypes. PRVG as well as PRVG*GM pointed towards HS612, HS507 and HPW430 for the better adaptable behavior and HS615 \& HS617 of low adaptability under irrigated timely sown 
Table 1: Parentage details and environmental conditions (2015-16)

\begin{tabular}{|c|c|c|c|c|c|}
\hline Genotype & Parentage & Locations & Latitude & Longitude & Altitude \\
\hline HPW 425 & (VL832/WH423) & Almora & $29^{\circ} 35^{\prime} \mathrm{N}$ & $79^{\circ} 39^{`} \mathrm{E}$ & 1610 \\
\hline VL2022 & (EC635640) & Khudwani & $33^{\circ} 70^{\prime} \mathrm{N}$ & $75^{\circ} 10^{\prime} \mathrm{E}$ & 1590 \\
\hline VL2019 & (RWP2002-2/SW89.3218//AGRI/NAC//VL905) & Malan & $32^{\circ} 08^{\prime} \mathrm{N}$ & $76^{\circ} 35^{\prime} \mathrm{E}$ & 846 \\
\hline HPW431 & (HPW42/HPW236) & Shimla & $31^{\circ} 10^{\prime} \mathrm{N}$ & $77^{\circ} 17^{\prime} \mathrm{E}$ & 2276 \\
\hline HPW430 & (HPW249/HPW211) & Bajaura & $31^{\circ} 50^{\prime} \mathrm{N}$ & $77^{\circ} 9^{\prime} \mathrm{E}$ & 1103.85 \\
\hline UP2953 & (WBLL1/KUKUNA//TACUPETO F2001/3/KIRITATI) & Wadura & $21^{\circ} 18^{\prime} \mathrm{N}$ & $77^{\circ} 41^{\prime} \mathrm{E}$ & 508 \\
\hline VL2024 & (W15.92/4/PASTOR//HXL7573/2*BAU/3/WBLL1/5/MUNAL) & & & & \\
\hline VL2023 & (ATTILA/3/WEAVER*2/TSC//WEAVER/4/ATTILA/PASTOR) & & & & \\
\hline HPW427 & (VL616/FLW3) & & & & \\
\hline HS616 & (SOKOLL/3/PASTOR//HXL7573/2*BAU) & & & & \\
\hline HS612 & $\begin{array}{l}\text { (SERI.1B*2/3KAUZ*2/BOW//KAUZ*2/5/CNO79/PF70354/ } \\
\text { MUS/3/PASTOR/4/BAV92) }\end{array}$ & & & & \\
\hline HPW429 & (ESWYT(2008)115/HPW211) & & & & \\
\hline HPW426 & (HPW155/HD29) & & & & \\
\hline UP2952 & (MILAN/S87230//BAV92*2/3/AKURI) & & & & \\
\hline HS615 & (BERKUT/HTG) & & & & \\
\hline HPW428 & (HPW155/HD29) & & & & \\
\hline HS613 & (WBM1587/VL824) & & & & \\
\hline HS614 & (HPW155/CHINESE LINE 14) & & & & \\
\hline HS617 & $\begin{array}{l}\text { (PASTOR/3/CROC-1/AE.SQUARROSA(224)//OPATA/4/ } \\
\text { BERKUT) }\end{array}$ & & & & \\
\hline VL2020 & (KLEIBER/2*FL80/DONSK.POLL/AKAW4006) & & & & \\
\hline VL2021 & (KLEIBER/2*FL80/DONSK.POLL/GW2000-18) & & & & \\
\hline HS618 & (BERKUT/HTG) & & & & \\
\hline HS507 & (KAUZ/MYNA/VUL//BUC/FLK/4/MILAN) & & & & \\
\hline VL907 & (DYBR 1982-83/842 ABVD 50/VW 9365//PBW 343) & & & & \\
\hline
\end{tabular}

Table 2: Parentage details and environmental conditions (2016-17)

\begin{tabular}{|c|c|c|c|c|c|}
\hline Genotype & Parentage & Locations & Latitude & Longitude & Altitude \\
\hline HPW 441 & (NAC/TH.AC//3*MIRLO/BUC/4/PASTOR) & Almora & $29^{\circ} 35^{\prime} \mathrm{N}$ & $79^{\circ} 39^{`} \mathrm{E}$ & 1610 \\
\hline HPW442 & (LONG291*2/PASTOR) & Khudwani & $33^{\circ} 70^{\prime} \mathrm{N}$ & $75^{\circ} 10^{\prime} \mathrm{E}$ & 1590 \\
\hline HPW443 & (PASTOR//HXL7573/2*BAU/3/SOKOLL/WBLL1) & Malan & $32^{\circ} 08^{\prime} \mathrm{N}$ & $76^{\circ} 35^{\prime} \mathrm{E}$ & 846 \\
\hline HPW 444 & $\begin{array}{l}\text { (AZAR2/4/CROC_1/AE.SQUARROSA (205)// } \\
\text { BORL95/3/2*MILAN/5/BERKUT) }\end{array}$ & Shimla & $31^{\circ} 10^{\prime} \mathrm{N}$ & $77^{\circ} 17^{\prime} \mathrm{E}$ & 2276 \\
\hline HPW445 & (PBW575/HPW251) & & & & \\
\hline HPW446 & (BOW/URES//KEA/3/SITE) & & & & \\
\hline HPW447 & (HPW266/HPW249) & & & & \\
\hline UP2991 & (SOKOLL/3/PASTOR//HXL7573/2*BAU/4/SOKOLL/WBLL1) & & & & \\
\hline HS631 & (WHEAR/VIVITSI//WHEAR) & & & & \\
\hline HS636 & (PASTOR//KAUZ/6/CNDO/R143//ENTE/MEX1-2/3/ & & & & \\
\hline HS635 & $\begin{array}{l}\text { AEGILOPSSQUARROSA(TAUS)/4/WEAVER/5/2*KAUZ) } \\
\text { (PFAU/MILAN/5/CHEN/AE.SQUARROSA(TAUS)//BCN/3/ } \\
\text { VEE\#7/BOW/4/PASTOR) }\end{array}$ & & & & \\
\hline HS632 & $(\mathrm{HS} 240 * 2 / \mathrm{FLW} 20(\mathrm{LR} 19) / / \mathrm{HS} 240 * 2 / \mathrm{FLW} 13(\mathrm{YR} 15)$ & & & & \\
\hline HS633 & (HS240*2/FLW20(LR19)//HS240*2/FLW13(YR15) & & & & \\
\hline HS634 & $\begin{array}{l}\text { (PBW343*2/KUKUNA/5/CNO79//PF73054/MUS/3/PASTOR/4/ } \\
\text { BAV92) }\end{array}$ & & & & \\
\hline HS637 & (PRL/2*PASTOR) & & & & \\
\hline UP2990 & (UP2744/WL711//PBW644) & & & & \\
\hline VL 2026 & (GW366/KS82W428/SWM75740//UP2739) & & & & \\
\hline VL 2027 & (RAJ4083/SKAUZ/HATUSA//VL900) & & & & \\
\hline VL 2025 & (LBPY04-1/RAJ4132//HS490) & & & & \\
\hline VL 2029 & (MUNAL\#1/FRANCOLIN\#1) & & & & \\
\hline VL 2028 & (FRANCOLIN\#1*2/MUU) & & & & \\
\hline VL2030 & (KA/NAC//TRCH/3/DANPHE\#1) & & & & \\
\hline VL 907 & (DYBR1982-8384ABVD50/VW9365//PBW343) & & & & \\
\hline HS 507 & (KAUZ/MYNA/VUL//BUC/FLK/4/MILAN) & & & & \\
\hline
\end{tabular}


Table 3: Adaptability measures of wheat genotypes as per BLUP(2015-16)

\begin{tabular}{|c|c|c|c|c|c|c|c|c|c|c|c|c|}
\hline & Almora & Kwani & Malan & Shimla & Mean & Sterr & GAI & PRVG & PRVG*GM & MHPVRG & MHPVRG*GM & MHVG \\
\hline HPW 425 & 17.87 & 42.85 & 23.49 & 24.68 & 27.22 & 6.25 & 25.81 & 1.07 & 26.90 & 1.06 & 26.56 & 24.63 \\
\hline VL2022 & 18.49 & 30.81 & 15.69 & 21.64 & 21.66 & 3.79 & 20.97 & 0.87 & 21.76 & 0.87 & 21.68 & 20.36 \\
\hline VL2019 & 21.93 & 26.10 & 23.29 & 23.71 & 23.76 & 1.00 & 23.71 & 1.00 & 25.03 & 0.96 & 24.05 & 23.67 \\
\hline HPW431 & 18.92 & 31.56 & 15.79 & 23.98 & 22.56 & 3.97 & 21.81 & 0.90 & 22.65 & 0.90 & 22.52 & 21.10 \\
\hline HPW430 & 20.31 & 43.89 & 20.82 & 27.63 & 28.16 & 6.35 & 26.76 & 1.11 & 27.76 & 1.10 & 27.68 & 25.60 \\
\hline UP2953 & 21.80 & 43.84 & 15.12 & 22.21 & 25.74 & 7.22 & 23.80 & 1.00 & 25.02 & 0.97 & 24.28 & 22.24 \\
\hline VL2024 & 19.43 & 35.09 & 23.98 & 27.72 & 26.56 & 3.82 & 25.95 & 1.08 & 27.00 & 1.07 & 26.75 & 25.36 \\
\hline VL2023 & 20.33 & 44.16 & 22.33 & 24.11 & 27.73 & 6.38 & 26.37 & 1.09 & 27.41 & 1.09 & 27.21 & 25.30 \\
\hline HPW427 & 13.29 & 33.81 & 18.46 & 25.12 & 22.67 & 5.12 & 21.37 & 0.89 & 22.37 & 0.87 & 21.87 & 20.12 \\
\hline HS616 & 18.52 & 39.83 & 17.98 & 28.62 & 26.24 & 5.95 & 24.82 & 1.03 & 25.81 & 1.02 & 25.60 & 23.58 \\
\hline HS612 & 24.66 & 37.95 & 26.90 & 27.41 & 29.23 & 3.43 & 28.82 & 1.20 & 30.04 & 1.18 & 29.66 & 28.46 \\
\hline HPW429 & 19.27 & 31.62 & 20.98 & 26.40 & 24.57 & 3.23 & 24.10 & 1.00 & 25.06 & 0.99 & 24.87 & 23.66 \\
\hline HS507 & 20.46 & 48.55 & 20.78 & 23.31 & 28.28 & 7.84 & 26.34 & 1.10 & 27.50 & 1.08 & 27.07 & 24.93 \\
\hline HPW426 & 17.53 & 42.59 & 19.16 & 27.34 & 26.66 & 6.62 & 25.01 & 1.04 & 26.03 & 1.03 & 25.77 & 23.63 \\
\hline UP2952 & 20.16 & 24.22 & 18.86 & 21.19 & 21.11 & 1.32 & 21.02 & 0.88 & 22.08 & 0.86 & 21.44 & 20.93 \\
\hline HS615 & 18.89 & 22.23 & 14.93 & 22.15 & 19.55 & 1.99 & 19.30 & 0.81 & 20.30 & 0.79 & 19.67 & 19.04 \\
\hline HPW428 & 15.02 & 45.63 & 18.41 & 25.14 & 26.05 & 7.92 & 23.73 & 1.00 & 24.94 & 0.97 & 24.23 & 21.91 \\
\hline HS613 & 20.08 & 36.93 & 21.14 & 24.68 & 25.71 & 4.47 & 24.94 & 1.03 & 25.85 & 1.03 & 25.82 & 24.29 \\
\hline HS614 & 16.02 & 39.40 & 16.10 & 22.67 & 23.55 & 6.36 & 21.91 & 0.91 & 22.83 & 0.90 & 22.56 & 20.62 \\
\hline HS617 & 19.23 & 28.38 & 14.73 & 22.51 & 21.21 & 3.32 & 20.62 & 0.86 & 21.49 & 0.85 & 21.23 & 20.04 \\
\hline VL907 & 20.75 & 27.11 & 22.25 & 24.15 & 23.56 & 1.58 & 23.45 & 0.98 & 24.60 & 0.96 & 23.95 & 23.33 \\
\hline VL2020 & 21.64 & 37.94 & 22.03 & 24.53 & 26.54 & 4.45 & 25.81 & 1.07 & 26.77 & 1.07 & 26.69 & 25.20 \\
\hline VL2021 & 21.96 & 43.72 & 16.79 & 28.99 & 27.86 & 6.75 & 26.14 & 1.09 & 27.30 & 1.07 & 26.83 & 24.62 \\
\hline HS618 & 19.15 & 38.42 & 16.92 & 26.17 & 25.16 & 5.59 & 23.89 & 0.99 & 24.82 & 0.98 & 24.67 & 22.78 \\
\hline
\end{tabular}

Table 4: Rank of wheat genotypes as per adaptability measures based on BLUP (2015-16)

\begin{tabular}{lllllllllllll}
\hline & Almora & Khudwani & Malan & Shimla Mean & Sterr GAI & PRVG & PRVG*GM MHPVRG MHPVRG*GM MHVG \\
\hline HPW 425 & 20 & 7 & 3 & 12 & 6 & 16 & 7 & 7 & 7 & 8 & 8 & 7 \\
VL2022 & 19 & 19 & 21 & 23 & 21 & 8 & 22 & 22 & 22 & 21 & 21 & 21 \\
VL2019 & 3 & 22 & 4 & 17 & 16 & 1 & 16 & 13 & 13 & 16 & 16 & 10 \\
HPW431 & 16 & 18 & 20 & 16 & 20 & 10 & 19 & 19 & 19 & 19 & 19 & 18 \\
HPW430 & 9 & 4 & 10 & 4 & 3 & 17 & 2 & 2 & 2 & 2 & 2 & 2 \\
UP2953 & 4 & 5 & 22 & 21 & 12 & 22 & 14 & 14 & 14 & 14 & 14 & 16 \\
VL2024 & 12 & 15 & 2 & 3 & 8 & 9 & 6 & 6 & 6 & 6 & 6 & 3 \\
VL2023 & 8 & 3 & 5 & 15 & 5 & 19 & 3 & 4 & 4 & 3 & 3 & 4 \\
HPW427 & 24 & 16 & 14 & 10 & 19 & 13 & 20 & 20 & 20 & 20 & 20 & 22 \\
HS616 & 18 & 9 & 16 & 2 & 10 & 15 & 11 & 11 & 11 & 11 & 11 & 13 \\
HS612 & 1 & 12 & 1 & 5 & 1 & 7 & 1 & 1 & 1 & 1 & 1 & 1 \\
HPW429 & 13 & 17 & 9 & 7 & 15 & 5 & 12 & 12 & 12 & 12 & 12 & 11 \\
HS507 & 7 & 1 & 11 & 18 & 2 & 23 & 4 & 3 & 3 & 4 & 4 & 6 \\
HPW426 & 21 & 8 & 12 & 6 & 7 & 20 & 9 & 9 & 9 & 10 & 10 & 12 \\
UP2952 & 10 & 23 & 13 & 24 & 23 & 2 & 21 & 21 & 21 & 22 & 22 & 19 \\
HS615 & 17 & 24 & 23 & 22 & 24 & 4 & 24 & 24 & 24 & 24 & 24 & 24 \\
HPW428 & 23 & 2 & 15 & 9 & 11 & 24 & 15 & 15 & 15 & 15 & 15 & 17 \\
HS613 & 11 & 14 & 8 & 11 & 13 & 12 & 10 & 10 & 10 & 9 & 9 & 9 \\
HS614 & 22 & 10 & 19 & 19 & 18 & 18 & 18 & 18 & 18 & 18 & 18 & 20 \\
HS617 & 14 & 20 & 24 & 20 & 22 & 6 & 23 & 23 & 23 & 23 & 23 & 23 \\
VL907 & 6 & 21 & 6 & 14 & 17 & 3 & 17 & 17 & 17 & 17 & 17 & 14 \\
VL2020 & 5 & 13 & 7 & 13 & 9 & 11 & 8 & 8 & 8 & 7 & 7 & 5 \\
VL2021 & 2 & 6 & 18 & 1 & 4 & 21 & 5 & 5 & 5 & 5 & 5 & 8 \\
HS618 & 15 & 11 & 17 & 8 & 14 & 14 & 13 & 16 & 16 & 13 & 13 & 15 \\
\hline
\end{tabular}


conditions for Northern Hills Zone. HS612, HS507 and HPW430 genotypes cited by analytic measures HMPRVG and HMPRVG*GM for high yield and better adaptability whereas HS615 \& HS617 for low of adaptation. An agreement has been maintained by analytic measures PRVG, MHVG, MHPRVG, GAI and average yield for the classification of wheat genotypes (Table 5). Differences among ranks of genotypes as per various locations highlighted cross over interactions effects for studied genotypes (Table 6).

\section{Biplot Analysis}

Two interaction principal components, accounted for $87.9 \%$ of total GxE interaction sum of squares in biplot analysis (Fig. 3). Stable performance of HS618, UP2953, HPW429, HS613, HPW431 and VL2022 genotypes would be as compared to HS612, VL2019, HPW428 and HS615 by virtue of their positions with respect to origin of biplot. Shimla location would be conducive for yield of genotypes vis-à-vis to Khudwani and Almora as positioned away from the origin. HPW429, HS613, VL2020, VL2024 had specific adaptations to Almora and Malan while HS616, HS618, HPW425, HPW426, HPW430, VL2023, VL2021, HPW426 for Shimla and Khudwani. Almora with Malan and Khudwani with Shimla expressed acute angles among rays therefore similar performance of genotypes would be expected. More over Almora had an obtuse angle with Khudwani i.e. HPW429 \& HS613 would not be suitable for Almora. Analytic measure standard error had maintained distance from others adaptability measures (Fig. 3).

\section{Second year (2017-18) based on BLUP}

\section{Analytic measures}

HPW447, HS631, HS632, VL2030 wheat genotypes were selected by average yield based on the BLUP values for possessing high yield with better adaptation and HS635, HS637 \& VL2028 for low yield \& specific adaptation. Harmonic mean identified HS631, HS632, VL2030, VL2025 for better adaptation along with suitability of HS635, VL2028 \& HPW444 for specific adaptations (Table 7). Least values of standard error associated with HPW446, HPW441, HS637 and VL907 genotypes for their consistent performance. HS631, HS632 VL2030,
HPW447 exhibited higher values of Geometric Adaptability Index while lower values by HS635, VL2028 \& HPW444. PRVG and PRVG*GM pointed towards HS631, HS632, HPW447, VL2030 for their better adaptation whereas HS635, VL2028 \& HPW444 for low adaptability. Analytic measures HMPRVG and HMPRVG* GM marked HS631, HS632, VL2030, VL2025 as of high yield and better adaptability as compared to HS635, VL2028 \& HPW444 wheat genotypes. An agreement had been observed among analytic measures PRVG, MHVG, MHPRVG, GAI and average yield for the classification of wheat genotypes (Table 7). Variations among ranks of genotypes as per various locations observed by adaptability measures to highlight presence of cross over interactions effects (Table 8).

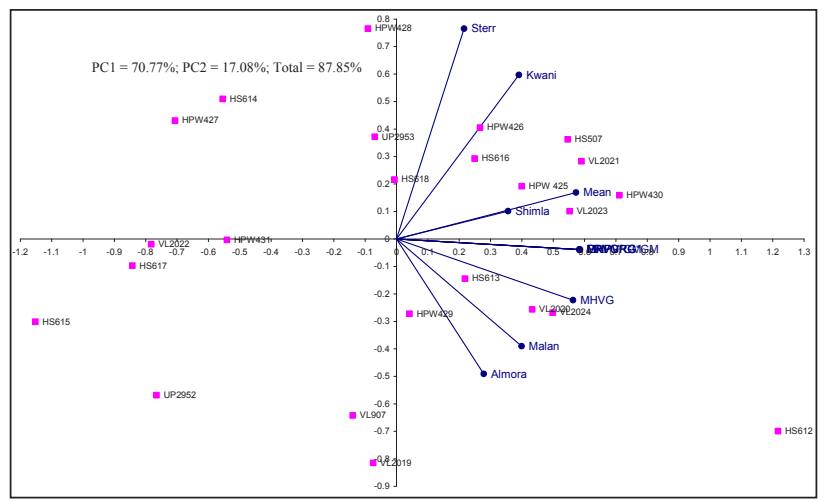

Fig. 3: Biplot analysis of genotypes vis-à-vis environments based on BLUE (2015-16)

\section{Biplot Analysis}

Total of $87.8 \%$ of G×E interaction sum of squares was explained by first two significant interaction principal components in biplot analysis (Fig. 4). Stable performance of HS618, UP2953, HPW429, HS613, HPW431 and VL2022 wheat genotypes were observed as compared to HS612, VL2019, HPW428 and HS615 by virtue of their positions with respect to origin of biplot. Shimla location would be conducive for yield of genotypes vis$\grave{a}$-vis to Khudwani and Almora as positioned away from the origin. HPW429, HS613, VL2020, VL2024 had specific adaptations to Almora and Malan while HS616, HS618, HPW425, HPW426, HPW430, VL2023, VL2021, HPW426 for Shimla and Khudwani. Acute angles among rays of Almora with Malan and Khudwani with Shimla expressed similar performance of genotypes. More over 
Table 5: Adaptability measures of wheat genotypes as per BLUE(2015-16)

\begin{tabular}{|c|c|c|c|c|c|c|c|c|c|c|c|c|}
\hline & Almora & Kwani & Malan & Shimla & Mean & Sterr & GAI & PRVG & PRVG*GM & MHPVRG & MHPVRG*GM & MHVG \\
\hline HPW 425 & 17.15 & 43.07 & 24.34 & 24.06 & 27.15 & 6.42 & 25.65 & 1.07 & 26.83 & 1.05 & 26.30 & 24.36 \\
\hline VL2022 & 18.46 & 30.73 & 15.31 & 20.98 & 21.37 & 3.84 & 20.66 & 0.86 & 21.45 & 0.85 & 21.35 & 20.03 \\
\hline VL2019 & 22.28 & 25.76 & 23.91 & 23.48 & 23.85 & 0.83 & 23.82 & 1.01 & 25.23 & 0.96 & 24.09 & 23.79 \\
\hline HPW431 & 19.02 & 31.41 & 15.23 & 24.14 & 22.45 & 4.04 & 21.65 & 0.90 & 22.52 & 0.89 & 22.33 & 20.89 \\
\hline HPW430 & 20.53 & 44.06 & 20.78 & 28.24 & 28.40 & 6.37 & 26.99 & 1.12 & 28.00 & 1.11 & 27.92 & 25.82 \\
\hline UP2953 & 22.91 & 44.27 & 14.30 & 21.25 & 25.68 & 7.47 & 23.56 & 1.00 & 24.95 & 0.95 & 23.85 & 21.83 \\
\hline VL2024 & 19.08 & 34.90 & 24.61 & 28.52 & 26.77 & 3.84 & 26.14 & 1.09 & 27.26 & 1.07 & 26.90 & 25.51 \\
\hline VL2023 & 20.43 & 44.48 & 22.81 & 23.32 & 27.76 & 6.48 & 26.37 & 1.10 & 27.45 & 1.08 & 27.17 & 25.29 \\
\hline HPW427 & 11.55 & 33.65 & 18.75 & 25.39 & 22.33 & 5.44 & 20.74 & 0.88 & 21.94 & 0.84 & 20.96 & 19.13 \\
\hline HS616 & 18.41 & 39.79 & 17.50 & 29.96 & 26.41 & 6.10 & 24.89 & 1.04 & 25.96 & 1.02 & 25.61 & 23.53 \\
\hline HS612 & 25.64 & 37.92 & 27.66 & 27.77 & 29.75 & 3.19 & 29.40 & 1.22 & 30.69 & 1.21 & 30.21 & 29.08 \\
\hline HPW429 & 19.07 & 31.35 & 21.17 & 27.07 & 24.67 & 3.23 & 24.20 & 1.00 & 25.18 & 1.00 & 24.94 & 23.74 \\
\hline HS507 & 20.77 & 49.09 & 21.02 & 22.15 & 28.25 & 8.03 & 26.25 & 1.10 & 27.47 & 1.07 & 26.90 & 24.80 \\
\hline HPW426 & 17.05 & 42.71 & 19.06 & 28.01 & 26.71 & 6.75 & 24.97 & 1.04 & 26.03 & 1.03 & 25.69 & 23.50 \\
\hline UP2952 & 20.33 & 23.91 & 18.98 & 20.43 & 20.91 & 1.21 & 20.84 & 0.88 & 21.93 & 0.85 & 21.22 & 20.76 \\
\hline HS615 & 18.97 & 21.80 & 14.38 & 22.11 & 19.31 & 2.07 & 19.04 & 0.80 & 20.07 & 0.77 & 19.36 & 18.74 \\
\hline HPW428 & 13.89 & 45.94 & 18.52 & 24.92 & 25.82 & 8.17 & 23.29 & 0.98 & 24.61 & 0.94 & 23.64 & 21.29 \\
\hline HS613 & 20.15 & 36.95 & 21.41 & 24.49 & 25.75 & 4.44 & 25.00 & 1.03 & 25.91 & 1.03 & 25.87 & 24.35 \\
\hline HS614 & 15.30 & 39.58 & 15.90 & 21.99 & 23.19 & 6.54 & 21.45 & 0.89 & 22.39 & 0.88 & 22.06 & 20.10 \\
\hline HS617 & 19.48 & 28.18 & 14.06 & 22.34 & 21.01 & 3.40 & 20.38 & 0.85 & 21.28 & 0.84 & 20.93 & 19.73 \\
\hline VL907 & 20.84 & 26.77 & 22.73 & 24.10 & 23.61 & 1.44 & 23.51 & 0.99 & 24.72 & 0.96 & 23.97 & 23.42 \\
\hline VL2020 & 22.10 & 38.02 & 22.34 & 24.18 & 26.66 & 4.40 & 25.96 & 1.08 & 26.95 & 1.07 & 26.82 & 25.37 \\
\hline VL2021 & 22.98 & 43.85 & 15.78 & 30.39 & 28.25 & 6.92 & 26.37 & 1.11 & 27.69 & 1.07 & 26.89 & 24.60 \\
\hline HS618 & 19.29 & 38.44 & 16.37 & 26.76 & 25.21 & 5.68 & 23.87 & 0.99 & 24.84 & 0.98 & 24.61 & 22.68 \\
\hline
\end{tabular}

Table 6: Rank of wheat genotypes as per adaptability measures based on BLUE(2015-16)

\begin{tabular}{|c|c|c|c|c|c|c|c|c|c|c|c|c|}
\hline & Almora & Kwani & Malan & Shimla & Mean & Sterr & GAI & PRVG & PRVG*GM & MHPVRG & MHPVRG*GM & MHVG \\
\hline HPW 425 & 20 & 7 & 3 & 15 & 6 & 17 & 8 & 8 & 8 & 8 & 8 & 8 \\
\hline VL2022 & 18 & 19 & 20 & 23 & 21 & 8 & 22 & 22 & 22 & 20 & 20 & 21 \\
\hline VL2019 & 4 & 22 & 4 & 16 & 16 & 1 & 14 & 12 & 12 & 14 & 14 & 10 \\
\hline HPW431 & 16 & 17 & 21 & 13 & 19 & 10 & 18 & 18 & 18 & 18 & 18 & 18 \\
\hline HPW430 & 8 & 5 & 11 & 4 & 2 & 16 & 2 & 2 & 2 & 2 & 2 & 2 \\
\hline UP2953 & 3 & 4 & 23 & 22 & 13 & 22 & 15 & 14 & 14 & 16 & 16 & 16 \\
\hline VL2024 & 14 & 15 & 2 & 3 & 7 & 9 & 6 & 6 & 6 & 4 & 4 & 3 \\
\hline VL2023 & 9 & 3 & 5 & 17 & 5 & 18 & 3 & 5 & 5 & 3 & 3 & 5 \\
\hline HPW427 & 24 & 16 & 14 & 9 & 20 & 13 & 21 & 20 & 20 & 22 & 22 & 23 \\
\hline HS616 & 19 & 9 & 16 & 2 & 10 & 15 & 11 & 10 & 10 & 11 & 11 & 12 \\
\hline HS612 & 1 & 13 & 1 & 6 & 1 & 5 & 1 & 1 & 1 & 1 & 1 & 1 \\
\hline HPW429 & 15 & 18 & 9 & 7 & 15 & 6 & 12 & 13 & 13 & 12 & 12 & 11 \\
\hline HS507 & 7 & 1 & 10 & 19 & 3 & 23 & 5 & 4 & 4 & 5 & 5 & 6 \\
\hline HPW426 & 21 & 8 & 12 & 5 & 8 & 20 & 10 & 9 & 9 & 10 & 10 & 13 \\
\hline UP2952 & 10 & 23 & 13 & 24 & 23 & 2 & 20 & 21 & 21 & 21 & 21 & 19 \\
\hline HS615 & 17 & 24 & 22 & 20 & 24 & 4 & 24 & 24 & 24 & 24 & 24 & 24 \\
\hline HPW428 & 23 & 2 & 15 & 10 & 11 & 24 & 17 & 17 & 17 & 17 & 17 & 17 \\
\hline HS613 & 11 & 14 & 8 & 11 & 12 & 12 & 9 & 11 & 11 & 9 & 9 & 9 \\
\hline HS614 & 22 & 10 & 18 & 21 & 18 & 19 & 19 & 19 & 19 & 19 & 19 & 20 \\
\hline HS617 & 12 & 20 & 24 & 18 & 22 & 7 & 23 & 23 & 23 & 23 & 23 & 22 \\
\hline VL907 & 6 & 21 & 6 & 14 & 17 & 3 & 16 & 16 & 16 & 15 & 15 & 14 \\
\hline VL2020 & 5 & 12 & 7 & 12 & 9 & 11 & 7 & 7 & 7 & 7 & 7 & 4 \\
\hline VL2021 & 2 & 6 & 19 & 1 & 4 & 21 & 4 & 3 & 3 & 6 & 6 & 7 \\
\hline HS618 & 13 & 11 & 17 & 8 & 14 & 14 & 13 & 15 & 15 & 13 & 13 & 15 \\
\hline
\end{tabular}


Almora had an obtuse angle with Khudwani i.e. HPW429 \& HS613 would not be suitable for Almora. Analytic measure standard error had maintained distance from others adaptability measures as observed from (Fig. 3).

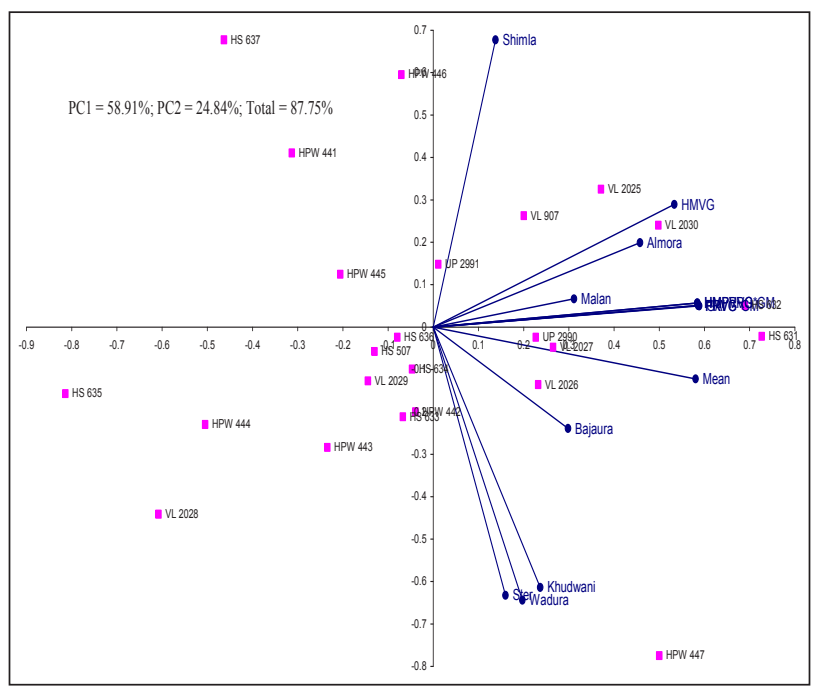

Fig. 4: Biplot analysis of genotypes vis-à-vis environments based on BLUP (2016-17)

\section{Second year (2017-18) based on BLUE}

\section{Analytic measures}

Wheat genotypes HS631, HPW447, HS632, VL2030 were identified by mean yield as per their BLUE values for high yield and better adaptation at the same time low yield \& specific adaptation of HS635, HS637 \& HPW444. Harmonic mean of genotypes values selected HS631, HS632, VL2030, VL2025 for better adaptation and specific adaptations of HS635, HPW444, VL2028 (Table 9). Consistent performance of HPW446, HPW445, VL907, HPW441 wheat genotypes would be judged by minimum values of standard error. Higher values of Geometric Adaptability Index exhibited by HS631, HS632 VL2030, HPW447 wheat genotypes for better adaptability and specific adaptations by HS635, HPW444 \&VL2028. PRVG and PRVG*GM measures expressed better adaptations of HS631, HS632, VL2030, HPW447 for locations of this zone. Analytic measures HMPRVG and HMPRVG*GM marked HS631, HS632, VL2030, VL2025 as of high yield and better adaptability as compared to HS635, VL2028 \& HPW444 wheat genotypes. Analytic measures of adaptability PRVG, MHVG, MHPRVG, GAI and average yield had maintained an agreement for the classification of wheat genotypes (Table 7). Differences among ranks of genotypes, as per various locations, observed by adaptability measures to highlight the presence of cross over interactions effects (Table 10). The ranking of genotypes through $\mathrm{HMRPGV}^{*} \mathrm{GY}$ was ideal and should be considered for the final recommendation of the best genotypes. This criterion of selection simultaneously considers stability, adaptability and grain yield of the genotypes grown in the experimental net environments.

\section{Biplot Analysis}

First two significant interaction principal components expressed $79.5 \%$ of total G×E interaction sum of squares in biplot analysis (Fig. 5). HS636, UP2991, HPW445, HS507, HPW442, HS634, HPW443 and VL2029 wheat genotypes observed near to origin as compared to HS635, HPW447 and HS637 genotypes in biplot presentations. Malan and Almora location would be conducive for yield of genotypes vis-à-vis to Shimla, Wadura and Khudwani as positioned away from the origin. HPW446, VL907, HS632, VL2025, VL2030 had specific adaptations to Almora and Shimla while HPW447, UP2990, HS631, HS633, VL2026, VL2027 for Wadura, Malan, Bajura and Khudwani. Acute angles between rays of Almora with Shimla and Khudwani with Wadura and Malan with Bajura expressed similar performance of genotypes. More over Shimla had an obtuse angle with Wadura i.e. VL907, HPW446, VL2025, VL2030 would not be suitable for Wadura location. Analytic measure standard error along with average yield observed in other quadrant with respect to others adaptability measures as seen from Fig. 5 .

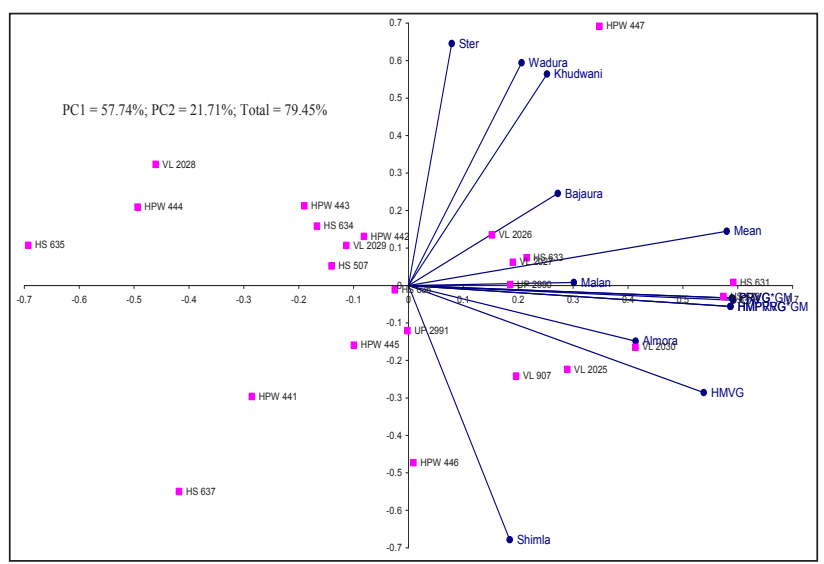

Fig. 5: Biplot analysis of genotypes vis-à-vis environments based on BLUE (2016-17) 
Table 7: Adaptability measures of wheat genotypes as per BLUP(2016-17)

\begin{tabular}{|c|c|c|c|c|c|c|c|c|c|c|c|c|c|c|}
\hline & $\frac{\pi}{\stackrel{0}{0}}$ & ص્ّ & 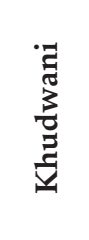 & $\frac{\tilde{J}}{\frac{\pi}{\tilde{J}}}$ & 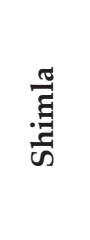 & 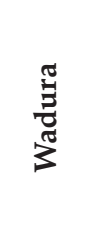 & $\underset{\Sigma}{\stackrel{\Xi}{\Sigma}}$ & ڤั & ষ্ডে & 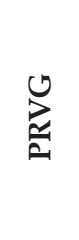 & $\begin{array}{l}\sum_{0} \\
\bigcup \\
\vdots \\
\vdots \\
\vdots\end{array}$ & 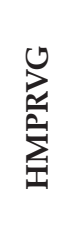 & 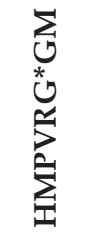 & $\sum^{U}$ \\
\hline HS 631 & 35.76 & 44.98 & 29.17 & 29.44 & 19.54 & 30.83 & 31.62 & 3.76 & 30.67 & 1.08 & 31.55 & 1.08 & 31.45 & 29.69 \\
\hline HS 634 & 34.01 & 39.48 & 27.18 & 29.10 & 15.54 & 29.90 & 29.20 & 3.58 & 28.11 & 0.99 & 28.91 & 0.99 & 28.83 & 26.82 \\
\hline HS 635 & 29.90 & 38.22 & 26.33 & 20.08 & 16.18 & 29.31 & 26.67 & 3.49 & 25.68 & 0.91 & 26.53 & 0.90 & 26.20 & 24.66 \\
\hline HS 636 & 34.29 & 37.34 & 29.07 & 23.64 & 18.12 & 30.89 & 28.89 & 3.15 & 28.10 & 0.99 & 28.93 & 0.98 & 28.78 & 27.25 \\
\hline HS 637 & 32.85 & 35.86 & 20.81 & 27.48 & 22.82 & 25.22 & 27.50 & 2.61 & 27.00 & 0.96 & 28.17 & 0.93 & 27.32 & 26.52 \\
\hline HPW 441 & 33.88 & 30.45 & 24.96 & 31.53 & 18.67 & 28.23 & 27.95 & 2.44 & 27.45 & 0.97 & 28.37 & 0.96 & 28.02 & 26.89 \\
\hline HPW 445 & 31.67 & 36.81 & 27.68 & 25.64 & 18.66 & 29.96 & 28.41 & 2.73 & 27.81 & 0.98 & 28.60 & 0.98 & 28.53 & 27.17 \\
\hline HPW 446 & 36.27 & 34.08 & 24.80 & 26.06 & 23.00 & 27.75 & 28.66 & 2.38 & 28.26 & 1.00 & 29.34 & 0.98 & 28.76 & 27.89 \\
\hline HPW 447 & 37.61 & 39.15 & 35.37 & 29.97 & 12.29 & 35.41 & 31.63 & 4.46 & 29.65 & 1.06 & 30.93 & 1.02 & 29.89 & 26.86 \\
\hline VL 2025 & 35.30 & 37.92 & 26.19 & 33.62 & 19.83 & 28.91 & 30.29 & 2.98 & 29.62 & 1.04 & 30.55 & 1.04 & 30.30 & 28.89 \\
\hline VL 2026 & 32.80 & 42.17 & 28.23 & 30.59 & 16.52 & 30.49 & 30.13 & 3.69 & 29.06 & 1.02 & 29.90 & 1.02 & 29.79 & 27.82 \\
\hline VL 2027 & 35.84 & 33.66 & 30.13 & 33.03 & 16.18 & 31.74 & 30.10 & 3.17 & 29.17 & 1.03 & 30.09 & 1.02 & 29.84 & 27.99 \\
\hline VL 2028 & 26.93 & 36.08 & 29.60 & 28.60 & 12.88 & 31.68 & 27.63 & 3.53 & 26.36 & 0.93 & 27.33 & 0.92 & 26.81 & 24.71 \\
\hline VL 2029 & 32.29 & 36.88 & 28.85 & 28.27 & 15.63 & 30.97 & 28.81 & 3.20 & 27.88 & 0.98 & 28.67 & 0.98 & 28.61 & 26.74 \\
\hline VL 2030 & 36.07 & 39.29 & 27.43 & 32.09 & 19.72 & 29.71 & 30.72 & 3.08 & 30.01 & 1.06 & 30.88 & 1.05 & 30.76 & 29.24 \\
\hline UP 2990 & 35.62 & 36.97 & 29.81 & 28.22 & 17.37 & 31.43 & 29.90 & 3.13 & 29.08 & 1.02 & 29.88 & 1.02 & 29.86 & 28.10 \\
\hline
\end{tabular}

Table 8: Ranks of wheat genotypes as per adaptability measures based on BLUP (2016-17)

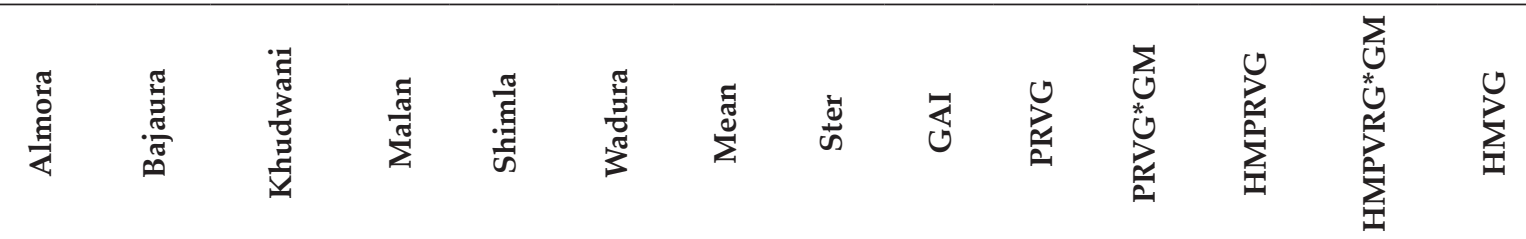

\begin{tabular}{lcccccccccccccc}
\hline HS 631 & 7 & 1 & 8 & 8 & 7 & 10 & 2 & 23 & 1 & 1 & 1 & 1 & 1 & 1 \\
HS 632 & 4 & 2 & 13 & 10 & 3 & 14 & 3 & 22 & 2 & 2 & 2 & 2 & 2 & 2 \\
HS 633 & 16 & 9 & 3 & 22 & 14 & 3 & 13 & 16 & 15 & 14 & 14 & 15 & 15 & 13 \\
HS 634 & 14 & 4 & 19 & 9 & 20 & 18 & 10 & 20 & 13 & 15 & 15 & 12 & 12 & 18 \\
HS 635 & 22 & 8 & 20 & 24 & 17 & 20 & 24 & 17 & 24 & 24 & 24 & 24 & 24 & 24 \\
HS 636 & 13 & 11 & 9 & 23 & 10 & 9 & 14 & 10 & 14 & 13 & 13 & 13 & 13 & 11 \\
HS 637 & 17 & 19 & 24 & 15 & 2 & 24 & 23 & 3 & 21 & 21 & 21 & 21 & 21 & 20 \\
HPW 441 & 15 & 24 & 22 & 5 & 8 & 22 & 20 & 2 & 20 & 20 & 20 & 20 & 20 & 15 \\
HPW 442 & 12 & 17 & 2 & 19 & 18 & 2 & 11 & 14 & 12 & 12 & 12 & 11 & 11 & 14 \\
HPW 443 & 23 & 14 & 7 & 4 & 22 & 7 & 17 & 15 & 19 & 19 & 19 & 19 & 19 & 21 \\
HPW 444 & 21 & 7 & 18 & 17 & 21 & 16 & 21 & 19 & 22 & 22 & 22 & 22 & 22 & 22 \\
HPW 445 & 20 & 16 & 16 & 20 & 9 & 17 & 19 & 5 & 18 & 18 & 18 & 18 & 18 & 12 \\
HPW 446 & 3 & 22 & 23 & 18 & 1 & 23 & 18 & 1 & 11 & 10 & 10 & 14 & 14 & 8 \\
HPW 447 & 1 & 6 & 1 & 7 & 24 & 1 & 1 & 24 & 4 & 3 & 3 & 5 & 5 & 17 \\
VL 2025 & 9 & 10 & 21 & 1 & 5 & 21 & 5 & 7 & 5 & 5 & 5 & 4 & 4 & 4
\end{tabular}




\begin{tabular}{lcccccccccccccc} 
VL 2026 & 18 & 3 & 14 & 6 & 15 & 12 & 6 & 21 & 9 & 8 & 8 & 8 & 8 & 9 \\
VL 2027 & 6 & 23 & 4 & 2 & 16 & 4 & 7 & 11 & 6 & 6 & 6 & 7 & 7 & 7 \\
VL 2028 & 24 & 18 & 6 & 11 & 23 & 5 & 22 & 18 & 23 & 23 & 23 & 23 & 23 & 23 \\
VL 2029 & 19 & 13 & 10 & 13 & 19 & 8 & 15 & 12 & 16 & 17 & 17 & 16 & 16 & 19 \\
VL 2030 & 5 & 5 & 17 & 3 & 6 & 19 & 4 & 8 & 3 & 4 & 4 & 3 & 3 & 3 \\
UP 2990 & 8 & 12 & 5 & 14 & 12 & 6 & 8 & 9 & 7 & 9 & 9 & 6 & 6 & 6 \\
UP 2991 & 11 & 20 & 15 & 12 & 11 & 15 & 12 & 6 & 10 & 11 & 11 & 10 & 10 & 10 \\
VL 907 & 2 & 21 & 12 & 16 & 4 & 13 & 9 & 4 & 8 & 7 & 7 & 9 & 9 & 5 \\
HS 507 & 10 & 15 & 11 & 21 & 13 & 11 & 16 & 13 & 17 & 16 & 16 & 17 & 17 & 16 \\
\hline
\end{tabular}

Table 9: Adaptability measures of wheat genotypes as per BLUE(2016-17)

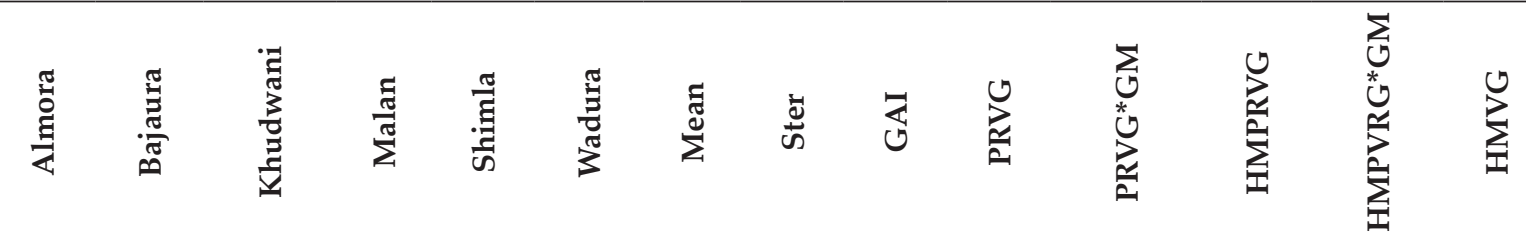

\begin{tabular}{|c|c|c|c|c|c|c|c|c|c|c|c|c|c|c|}
\hline HS 631 & 35.53 & 46.07 & 30.47 & 29.60 & 20.36 & 29.69 & 31.95 & 3.79 & 31.03 & 1.09 & 31.96 & 1.09 & 31.78 & 30.11 \\
\hline HS 632 & 36.17 & 45.80 & 28.64 & 29.11 & 20.89 & 30.42 & 31.84 & 3.76 & 30.94 & 1.09 & 31.88 & 1.08 & 31.68 & 30.08 \\
\hline HS 633 & 33.49 & 38.21 & 30.96 & 29.20 & 17.23 & 32.07 & 30.19 & 3.15 & 29.35 & 1.03 & 30.17 & 1.03 & 30.12 & 28.33 \\
\hline HS 634 & 35.00 & 39.91 & 25.59 & 29.29 & 13.75 & 29.70 & 28.87 & 4.00 & 27.45 & 0.97 & 28.33 & 0.96 & 28.04 & 25.72 \\
\hline HS 635 & 29.26 & 38.48 & 25.96 & 18.93 & 15.89 & 29.17 & 26.28 & 3.62 & 25.21 & 0.89 & 26.11 & 0.88 & 25.64 & 24.12 \\
\hline HS 636 & 33.97 & 37.23 & 29.53 & 22.95 & 18.93 & 31.49 & 29.02 & 3.08 & 28.27 & 1.00 & 29.16 & 0.99 & 28.90 & 27.48 \\
\hline HS 637 & 32.82 & 35.89 & 20.40 & 27.46 & 22.86 & 23.37 & 27.13 & 2.74 & 26.58 & 0.95 & 27.81 & 0.92 & 26.81 & 26.05 \\
\hline HPW 441 & 34.43 & 29.46 & 23.98 & 32.10 & 18.04 & 28.14 & 27.69 & 2.65 & 27.10 & 0.96 & 28.05 & 0.94 & 27.62 & 26.45 \\
\hline HPW 442 & 35.15 & 35.98 & 31.86 & 25.45 & 15.36 & 30.07 & 28.98 & 3.43 & 27.92 & 0.98 & 28.78 & 0.98 & 28.57 & 26.63 \\
\hline HPW 443 & 28.27 & 36.79 & 29.82 & 32.10 & 14.02 & 31.01 & 28.67 & 3.46 & 27.50 & 0.97 & 28.46 & 0.96 & 28.03 & 25.99 \\
\hline HPW 444 & 30.29 & 38.84 & 26.24 & 26.03 & 13.04 & 29.81 & 27.37 & 3.77 & 26.05 & 0.92 & 26.89 & 0.91 & 26.61 & 24.44 \\
\hline HPW 445 & 30.51 & 36.70 & 28.50 & 25.27 & 20.00 & 30.28 & 28.54 & 2.51 & 28.06 & 0.99 & 28.92 & 0.98 & 28.72 & 27.56 \\
\hline HPW 446 & 36.40 & 33.57 & 23.78 & 25.76 & 24.29 & 29.49 & 28.88 & 2.33 & 28.50 & 1.01 & 29.70 & 0.99 & 28.89 & 28.14 \\
\hline HPW 447 & 38.73 & 39.11 & 35.16 & 30.18 & 11.34 & 36.92 & 31.91 & 4.73 & 29.60 & 1.06 & 31.07 & 1.01 & 29.60 & 26.29 \\
\hline VL 2025 & 35.67 & 38.04 & 25.47 & 34.46 & 19.82 & 29.04 & 30.42 & 3.10 & 29.69 & 1.05 & 30.65 & 1.04 & 30.35 & 28.92 \\
\hline VL 2026 & 32.66 & 42.95 & 27.93 & 30.98 & 16.16 & 30.30 & 30.16 & 3.85 & 28.99 & 1.02 & 29.86 & 1.01 & 29.70 & 27.67 \\
\hline VL 2027 & 36.49 & 32.95 & 30.41 & 33.75 & 15.71 & 31.59 & 30.15 & 3.30 & 29.14 & 1.03 & 30.10 & 1.02 & 29.76 & 27.83 \\
\hline VL 2028 & 25.21 & 35.89 & 30.23 & 28.71 & 13.13 & 32.01 & 27.53 & 3.53 & 26.29 & 0.93 & 27.30 & 0.91 & 26.68 & 24.70 \\
\hline VL 2029 & 32.05 & 36.79 & 28.33 & 28.30 & 15.45 & 31.89 & 28.80 & 3.24 & 27.84 & 0.98 & 28.63 & 0.97 & 28.55 & 26.65 \\
\hline VL 2030 & 36.43 & 39.55 & 26.80 & 32.68 & 20.00 & 30.36 & 30.97 & 3.13 & 30.25 & 1.06 & 31.14 & 1.06 & 30.99 & 29.47 \\
\hline UP 2990 & 35.88 & 36.79 & 30.51 & 28.21 & 17.50 & 31.10 & 30.00 & 3.11 & 29.19 & 1.02 & 29.99 & 1.02 & 29.96 & 28.22 \\
\hline UP 2991 & 35.19 & 35.18 & 27.63 & 28.62 & 17.77 & 30.00 & 29.06 & 2.87 & 28.37 & 1.00 & 29.15 & 0.99 & 29.12 & 27.57 \\
\hline VL 907 & 37.35 & 33.57 & 29.42 & 27.32 & 20.71 & 30.32 & 29.78 & 2.53 & 29.30 & 1.03 & 30.22 & 1.02 & 29.97 & 28.79 \\
\hline HS 507 & 35.82 & 36.70 & 28.34 & 23.66 & 16.52 & 30.71 & 28.63 & 3.42 & 27.65 & 0.97 & 28.48 & 0.97 & 28.32 & 26.57 \\
\hline
\end{tabular}

Table 10: Rank of wheat genotypes as per adaptability measures based on BLUE (2016-17)

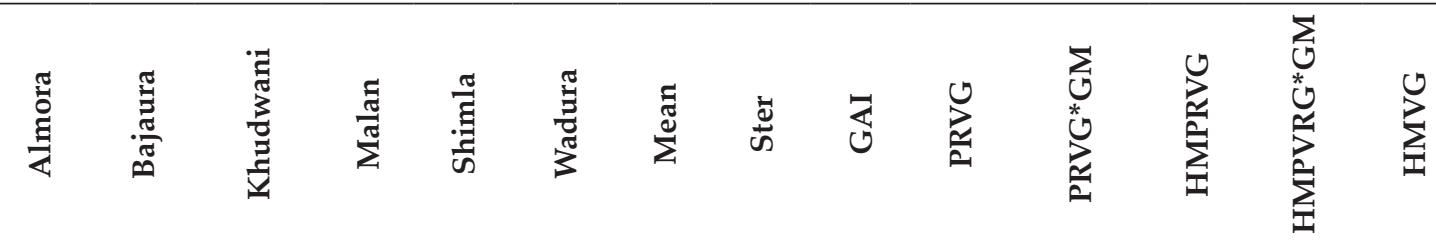

\begin{tabular}{lcccccccccccccc}
\hline HS 631 & 10 & 1 & 5 & 8 & 5 & 19 & 1 & 21 & 1 & 1 & 1 & 1 & 1 & 1 \\
HS 632 & 6 & 2 & 11 & 11 & 3 & 10 & 3 & 19 & 2 & 2 & 2 & 2 & 2 & 2 \\
HS 633 & 16 & 9 & 3 & 10 & 13 & 2 & 6 & 11 & 6 & 7 & 7 & 5 & 5 & 6 \\
HS 634 & 13 & 4 & 20 & 9 & 21 & 18 & 15 & 23 & 19 & 19 & 19 & 18 & 18 & 21 \\
HS 635 & 22 & 8 & 19 & 24 & 16 & 21 & 24 & 18 & 24 & 24 & 24 & 24 & 24 & 24
\end{tabular}




\begin{tabular}{lcccccccccccccc}
\hline HS 636 & 15 & 11 & 9 & 23 & 9 & 6 & 12 & 7 & 13 & 12 & 12 & 12 & 12 & 13 \\
HS 637 & 17 & 18 & 24 & 16 & 2 & 24 & 23 & 5 & 21 & 21 & 21 & 21 & 21 & 19 \\
HPW 441 & 14 & 24 & 22 & 4 & 10 & 23 & 20 & 4 & 20 & 20 & 20 & 20 & 20 & 17 \\
HPW 442 & 12 & 17 & 2 & 20 & 19 & 15 & 13 & 15 & 15 & 15 & 15 & 15 & 15 & 15 \\
HPW 443 & 23 & 13 & 8 & 4 & 20 & 8 & 17 & 16 & 18 & 18 & 18 & 19 & 19 & 20 \\
HPW 444 & 21 & 7 & 18 & 18 & 23 & 17 & 22 & 20 & 23 & 23 & 23 & 23 & 23 & 23 \\
HPW 445 & 20 & 15 & 12 & 21 & 6 & 14 & 19 & 2 & 14 & 14 & 14 & 14 & 14 & 12 \\
HPW 446 & 5 & 21 & 23 & 19 & 1 & 20 & 14 & 1 & 11 & 11 & 11 & 13 & 13 & 8 \\
HPW 447 & 1 & 6 & 1 & 7 & 24 & 1 & 2 & 24 & 5 & 4 & 4 & 10 & 10 & 18 \\
VL 2025 & 9 & 10 & 21 & 1 & 8 & 22 & 5 & 8 & 4 & 5 & 5 & 4 & 4 & 4 \\
VL 2026 & 18 & 3 & 15 & 6 & 15 & 13 & 7 & 22 & 10 & 10 & 10 & 9 & 9 & 10 \\
VL 2027 & 3 & 23 & 6 & 2 & 17 & 5 & 8 & 13 & 9 & 8 & 8 & 8 & 8 & 9 \\
VL 2028 & 24 & 18 & 7 & 12 & 22 & 3 & 21 & 17 & 22 & 22 & 22 & 22 & 22 & 22 \\
VL 2029 & 19 & 13 & 14 & 14 & 18 & 4 & 16 & 12 & 16 & 16 & 16 & 16 & 16 & 14 \\
VL 2030 & 4 & 5 & 17 & 3 & 6 & 11 & 4 & 10 & 3 & 3 & 3 & 3 & 3 & 3 \\
UP 2990 & 7 & 12 & 4 & 15 & 12 & 7 & 9 & 9 & 8 & 9 & 9 & 7 & 7 & 7 \\
UP 2991 & 11 & 20 & 16 & 13 & 11 & 16 & 11 & 6 & 12 & 13 & 13 & 11 & 11 & 11 \\
VL 907 & 2 & 21 & 10 & 17 & 4 & 12 & 10 & 3 & 7 & 6 & 6 & 6 & 6 & 5 \\
HS 507 & 8 & 15 & 13 & 22 & 14 & 9 & 18 & 14 & 17 & 17 & 17 & 17 & 17 & 16 \\
\hline
\end{tabular}

\section{CONCLUSION}

Classification of wheat genotypes as per their adaptability by analytic measures based on BLUP by exploiting REML procedure was more efficient than that by BLUE (Smith and Cullis 2018). Biplot analysis showed that more of $\mathrm{G} \times \mathrm{E}$ interactions sum of squares was explained by first two principal components as compared to accounted by BLUE. There was no overall difference between analytic measures as far as adaptability of genotypes is concerned either based on Harmonic or Geometric means as compared to usual mean yield of genotypes. Genotype classifications by BLUP/ REML were superior to that of by BLUE for both years, despite the presence of cross over genotype x environment interactions (Kleinknecht et al. 2011).

\section{ACKNOWLEDGEMENTS}

Guidance and financial support extended by Dr RP Singh, CIMMYT Mexico acknowledged by the authors. Efforts of staff, working at various coordinated centers of wheat, are highly appreciated for field evaluation wheat genotypes.

\section{REFERENCES}

Baretta, D., Nardino, M., Carvalho, I.R., Oliveira, A.C., Souza, V.Q. and Maia, L.C. 2016. Performance of Maize Genotypes of Rio Grande do Sul using Mixed Models. Cientifica, 44: 403-411.

Crespo-Herrera, L.A., Crossa J., Huerta-Espino J., Autrique E., Mondal, S. and Velu, G. 2017. Genetic yield gains in
CIMMYT's International Elite Spring Wheat Yield Trials by modeling the genotype $\times$ environment interaction. Crop Sci., 57: 789-801.

Cullis, B.R., Jefferson P., Thompson R. and Smith A.B. 2014. Factor analytic and reduced animal models for the investigation of additive genotype by environment interaction in outcrossing plant species with application to a pinus radiata breeding program. Theor. Appl. Genet., 127: 2193-2210.

de Pelegrin, A.J., Carvalho, I.R., Nunes, A.C.P., Demari, G.H., Szareski, V.J., Barbosa, M.H., da Rosa, T.C., Ferrari, M., Nardino, M., dos Santos, O.P., de Resende, M.D.V., de Souza, V.Q., de Oliveira, A.C. and da Maia, L.C. 2017. Adaptability, Stability and Multivariate Selection by Mixed Models. American Journal of Plant Sciences, 8: 33243337.

Diego, B., Maicon N., Ivan R.C., Antonio C. de O., Velci Q. de S. and Luciano C. da M. .2016. Performance of maize genotypes of Rio Grande do Sul using mixed models. Científica, Jaboticabal, 44(3): 403-411.

Elesandro, B., Giovani B., Lindolfo S., Leomar G. W., Thiago D., Matheus G.S. and Sergio, V.M. 2017. Statistical methods to study adaptability and stability of wheat genotypes. Bragantia, Campinas, 76(1): 1-10.

Friesen, L.F., Brule-Babel A.L., Crow, G.H. and Rothenburger, P.A. 2016. Mixed model and stability analysis of spring wheat genotype yield evaluation data from Manitoba, Canada. Can. J. Plant Sci., 96(2): 305-320.

Gogel, B.J., Smith A.B. and Cullis, B.R . 2018. Comparison of a one and two-stage mixed model analysis of Australia's National Variety Trial Southern Region wheat data. Euphytica., 214(2): 44-64.

Kleinknecht, K., Laidig, F., Piepho, H.P. and Möhring, J. 2011. Best linear unbiased prediction (BLUP): Is it beneficial in official variety performance trials? Biuletyn Oceny Odmian, 33: 21-33. 
Mendes, F.F., Guimarães L.J.M., Souza J.C., Guimarães, P.E.O., Pacheco, C.A.P., Machado, J.R. de A, Meirelles, W.F., Silva, A.R. da and Parentoni, S. 2012. Adaptability and stability of maize varieties using mixed model methodology. Crop Breeding and Applied Biotechnology, 12(2): 111-117.

Moiana, L.D., Vidigal Filho, P.D., Gonçalves-Vidigal, M.C. and Maleia, M.P. 2014. Application of mixed models for the assessment genotype and environment interactions in cotton (Gossypium hirsutum) cultivars in Mozambique. Afr. J. Biotechnology, 13: 1985-1991.

Mohammadi, R. and Amri, A. 2008. Comparison of parametric and non-parametric methods for selecting stable and adapted durum wheat genotypes in variable environments. Euphytica, 159: 419-432.

Nuvunga, J.J., Oliveira, L.A., Silva, C.P., Pamplona, A.K.A., Silva, A.Q., Moura, E.G., Maleia, M.P. and Balestre, M. 2018. Adaptability and stability of cotton cultivars (Gossypium hirsutum L. race latifolium $H$.) using factor analytic model. Genet. Mol. Res., 17(1): 1-10.

Oliveira, I.J. de, Atroch, A.L., Dias, M.C., Guimarães, L.J. and Paulo, E. de O.G. 2017. Selection of corn cultivars for yield, stability and adaptability in the state of Amazonas, BrazilPesq. Agropec. Bras., Brasília, 52(6): 455-463.

Piepho, H.P., Möhring, J., Melchinger, A.E. and Büchse, A. 2008. BLUP for phenotypic selection in plant breeding and variety testing. Euphytica, 161: 209-228.

Resende, M.D.V. and Duarte, J.B. 2007. Precision and quality control in variety trials. Pesquisa Agropecuária Tropical, 37: 182-194.
Santos, E.A., Viana, A.P., Freitas, J.C.O., Rodrigues, D.L., Tavares, R.F., Paiva, C.L. and Souza, M.M. 2015. Genotype selection by REML/BLUP methodology in a segregating population from na interspecific Passiflora spp. crossing. Euphytica, 204(1): 1-11.

Silva, P. da., Bisognin D.A., Locatelli A.B. and Storck, L. 2014. Adaptability and stability of corn hybrids grown for high grain yield. Acta Scientiarum. Agronomy, 36: 175-181.

Smith, A.B. and Cullis B.R. 2018. Plant breeding selection tools built on factor analytic mixed models for multienvironment trial data. Euphytica, 214(8): 143-161.

Tadege, M.B., Utta, H.Z. and Aga, A.A. 2014. Association of statistical methods used to explore genotype $x$ environment interaction (GEI) and cultivar stability. African Journal of Agricultural Research, 9: 2231-2237.

Torres, F.E., Teodoro, P.E., Sagrillo, E., Ceccon, G. and Correa, A.M. 2015. Genotype $\times$ Environment Interaction in Semiprostrade Cowpea Genotypes via Mixed Models. Bragantia, 74: 255-260.

Verardi, C.K., Resende M.D.Z.V., Costa, R.B. and Gonçalves P.S. 2009. Adaptabilidade e estabilidade da produção de borracha e seleção em progênies de seringueira. Pesquisa Agropecuária Brasileira, 44: 1277-1282.

Yan, W. and Kang, M.S. 2003. GGE Biplot Analysis: A Graphical Tool for Breeders, Geneticists, and Agronomists. CRC Press. Boca Raton, FL. 